\title{
Sputum eosinophilia does not correlate with exacerbations in asthmatic children treated with mepolizumab
}

Genoveva del-Rio Camacho ${ }^{1}$, Rebeca Lopez ${ }^{1}$, Mar Fernandez-Nieto ${ }^{2}$, Jose Antonio Cañas ${ }^{2}$, and María Jesús Rodríguez-Nieto ${ }^{1}$

${ }^{1}$ Hospital Universitario Fundación Jiménez Díaz

${ }^{2}$ Hospital Universitario Fundacion Jimenez Diaz

March 27, 2021

\section{TITLE PAGE}

TITLE : Sputum eosinophilia does not correlate with exacerbations in asthmatic children treated with mepolizumab.

\section{AUTHORS:}

del-Río Camacho G ${ }^{1}$ (MD, PhD) (ORCID: 0000-0002-9401-1864), López-Gómez $\mathrm{R}^{1}$ (MD), FernándezNieto $\mathrm{M}^{2}(\mathrm{MD})$, Cañas $\mathrm{JA}^{3}(\mathrm{PhD})$ (ORCID: 0000-0002-0460-2813), Rodríguez-Nieto $\mathrm{MJ}^{4}$ (MD, PhD)(ORCID: 0000-0003-4195-9528)

Departments of Pediatrics ${ }^{1}$, Allergy ${ }^{2}$, Immunology ${ }^{3}$ and Pneumology ${ }^{4}$, University Hospital Fundación Jiménez Díaz, Madrid (Spain)

\section{CORRESPONDENCE AUTHOR:}

Genoveva del-Río Camacho (MD, PhD)

Pediatrics department, University Hospital Fundación Jiménez Díaz

Avda. Reyes Católicos 2; 28040 Madrid (Spain)

Telephone number: 0034627805136

vevirio@hotmail.com

KEY WORDS: sputum eosinophilia, mepolizumab, asthma, children

FINANTIAL DISCLOSURE:

Del-Río Camacho G, Fernández-Nieto M, and Rodríguez-Nieto MJ, have recived lecture fees from GSK.

ABREVIATED TITLE: Sputum eosinophilia in children with mepolizumab

SPUTUM EOSINOPHILIA DOES NOT CORRELATE WITH EXACERBATIONS IN ASTHMATIC CHILDREN TREATED WITH MEPOLIZUMAB

To the Editor,

According to the latest guidelines on asthma in children and adolescents, step 5-6 of treatment recommends biologic therapies to control the disease. Initially, omalizumab was the only such drug used in children; now, however, other biologics such as mepolizumab that target IL- 5 are feasible for children over 6 years of age diagnosed with non-allergic eosinophilic asthma. 


\section{CASE REPORT}

A 12-year-old girl with severe, poorly controlled asthma was considered for treatment with omalizumab after 1 year of follow-up ( $\operatorname{lgE} 129$ ). Her lung function was studied at the time of diagnosis, including baseline spirometry (FEV1: 1.12 (56.9\%, Zs -3.8), FVC: 1.64 (71.2\%, Zs -2.63), FEV1/FVC ratio 58.43\% (Zs 2.64), MMEF $75 / 25: 0.54(20.8 \%, \mathrm{Zs}-4.17))$. She had a positive bronchodilation test (FEV1 $+16.5 \%)$ and FeNO levels of $121 \mathrm{ppb}$. A skin prick test was positive for olive and grass, though no clinical correlation was found. Omalizumab was maintained over 2 years, with no clinical improvement; during this time, the patient experienced exacerbations for which she was treated with oral steroids, and on one occasion she required hospital admission. Her lung function failed to improve, except when she was tested after 1 month of mistakenly continuing to take oral steroids (FEV1 2.52 (98.8\%, Zs -0.73), FVC $3.39(113.3 \%$, Zs +0.76), FEV1/FVC ratio 74.21 (Zs -2.10), MMEF 2.01 (62.2\%, Zs -2.03)).

At age 14.5 years, with no real clinical improvement or improved lung function (FEV1 1.92 (70.2\%, Zs -3.02), FVC 2.77 (86.2\%, Zs -1.54), FEV1/FVC ratio 69.19\% (Zs -2.59), MMEF 1.05 (30.7\%, Zs -3.80), her therapy was changed to mepolizumab (100 mg/4 weeks). Weight gain might also contribute to suboptimal control, no others comorbidities had been observed. At that moment she presented blood $\left(900 / \mathrm{mm}^{3}\right)$ and sputum eosinophilia (8.4\%). In the first of 2 years that have passed since this modification, sputum samples were obtained at baseline, 1, 2, 6, and 12 months; additionally, blood eosinophils were measured at 12 months. Results of spirometry testing normalized after 6 months (FEV1 107.4\% (Zs - 0.47 ), FVC 108.2\% (Zs +0.02), FEV1/FVC ratio 84.27\% (Zs -0.89), MMEF 2.27 (66.4T, Zs -1.97), though these results have varied (Fig.1). Both blood and sputum eosinophils evidenced marked changes, reducing to $100 / \mathrm{mm}^{3}$ and $0 \%$, respectively (Fig.1). However, her asthma remains uncontrolled. In the first year after mepolizumab was prescribed, she had 3 exacerbations, requiring oral corticosteroid treatment and hospital admission. Some of the sputum samples were collected at the time of the exacerbations, though these revealed no increase in the eosinophil levels (Fig.1). Her Asthma Control Test also showed poor control (13-20/25). She has not developed exacerbations during this, the second year, though as of the time of writing she requires both highdose inhaled corticosteroid (fluticasone $1000 \mathrm{mcg} /$ day), long-acting $\beta 2$-agonist, and montelukast to control her symptoms.

\section{DISCUSSION}

Treatment with mepolizumab has shown clinical improvement in patients with severe eosinophilic asthma, as reflected in a Cochrane review with 1707 patients aged over 12 years, in whom a reduction in exacerbations was observed (53\% (95\% CI 37-65) as well as an increase in baseline FEV1 value ( $\mathrm{p}>0.03)^{1}$. However, not all studies report these same findings, as some patients do not achieve optimal control ${ }^{2-4}$, which may be the case in our patient.

Most publications show decreased blood and sputum eosinophils in adult patients after treatment with mepolizumab ${ }^{3,5}$. This finding is controversial, however, and there is not always a correlation between the clinical picture and eosinophil levels. In a clinical trial with mepolizumab versus placebo ${ }^{5}$, patients in the placebo group continued to have exacerbations with sputum eosinophilia at the time of exacerbation, whereas only one patient on mepolizumab developed an exacerbation; this episode was not associated with sputum eosinophilia, as opposed to our patient during the first year, when sputum eosinophilia remained $0 \%$ despite clinical exacerbations. Conversely, cases of good clinical control have been described despite having no substantial decrease in eosinophils in sputum ${ }^{6}$. This seems to indicate that eosinophils are either not the only factor behind the inflammatory cascade in asthmatic exacerbations or that they are not indicative of clinical course. In our case, it seems that once mepolizumab was administered, sputum eosinophils were reduced to zero and did not increase, even in the case of exacerbations.

No previous studies in children report a decrease in eosinophils in sputum, though one group ${ }^{7}$ has found higher blood eosinophils among children, thus resembling findings in adults. Research in adults ${ }^{2}$ indicates that blood eosinophil monitoring is not useful in patients who do not achieve good asthma control after treatment with mepolizumab. In light of our findings, it appears that the same holds for sputum eosinophil 
count.

In children older than 6 years of age with poorly controlled severe asthma, non-responders to omalizumab (especially with eosinophilia but without a clear allergic pattern), modification or direct prescription of mepolizumab should be considered. Our patient has improved moderately after treatment with this drug and has achieved normalized lung function, though she continues to present exacerbations. Findings from this case suggest that sputum eosinophil count does not reflect the likelihood of exacerbations, leading us to call into question the usefulness of this marker.

\section{ACKNOWLEDGMENTS:}

We thank Oliver Shaw for editorial assistance.

\section{REFERENCES}

1. Powell C, Milan SJ, Dwan K, Bax L, Walters N. Mepolizumab versus placebo for asthma. Cochrane Database Syst Rev. 27 de julio de 2015;(7):CD010834.

2. Mukherjee M, Forero DF, Tran S, Boulay M-E, Bertrand M, Bhalla A, et al. Suboptimal treatment response to anti-IL-5 monoclonal antibodies in severe eosinophilic asthmatics with airway autoimmune phenomena. Eur Respir J. octubre de 2020;56(4).

3. Flood-Page P, Swenson C, Faiferman I, Matthews J, Williams M, Brannick L, et al. A study to evaluate safety and efficacy of mepolizumab in patients with moderate persistent asthma. Am J Respir Crit Care Med. 1 de diciembre de 2007;176(11):1062-71.

4. Kips JC, O'Connor BJ, Langley SJ, Woodcock A, Kerstjens HAM, Postma DS, et al. Effect of SCH55700, a humanized anti-human interleukin- 5 antibody, in severe persistent asthma: a pilot study. Am J Respir Crit Care Med. 15 de junio de 2003;167(12):1655-9.

5. Nair P, Pizzichini MMM, Kjarsgaard M, Inman MD, Efthimiadis A, Pizzichini E, et al. Mepolizumab for prednisone-dependent asthma with sputum eosinophilia. N Engl J Med. 5 de marzo de 2009;360(10):985-93.

6. Koya T, Kimura Y, Hayashi M, Hasegawa T, Kikuchi T. The impact of mepolizumab on the sputum level of eosinophil-derived protein in three cases of severe asthma. Allergol Int. octubre de 2020;69(4):639-41.

7. Gupta A, Pouliquen I, Austin D, Price RG, Kempsford R, Steinfeld J, et al. Subcutaneous mepolizumab in children aged 6 to 11 years with severe eosinophilic asthma. Pediatr Pulmonol. diciembre de 2019;54(12):1957-67.

Figure 1: Blood eosinophils at baseline (change to mepolizumab) and 12-month treatment. Sputum eosinophils at baseline, 1, 2, 6, and 12 months. All FEV1 (\%) values.

OS $=$ oral steroids. 


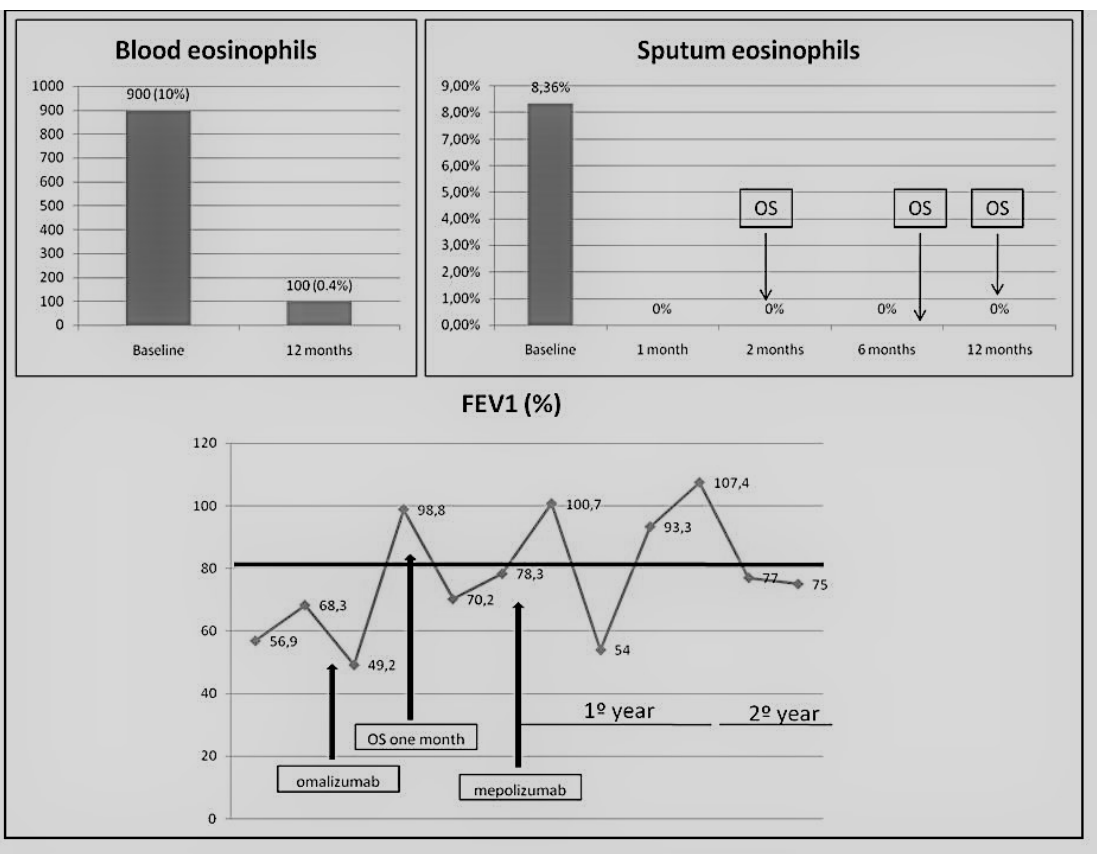

